REVISTA BRASILEIRA DE ANÁLISE DO COMPORTAMENTO / BRAZILIAN JOURNAL OF BEHAVIOR ANALYSIS, 2016, Vol. 12, № 1, $23-32$.

\title{
INTEGRAÇÃO DOS REPERTÓRIOS DE FALANTE-OUVINTE VIA INSTRUÇÃO COM EXEMPLARES MÚLTIPLOS EM CRIANÇAS IMPLANTADAS COCLEARES
}

\section{TEACHING INTEGRATION OF SPEAKER-LISTENER REPERTOIRES VIA MULTIPLE-EXEMPLAR INSTRUCTION IN COCHLEAR IMPLANTED CHILDREN}

\author{
FABIANE DA SILVA PEREIRA \\ GRAUBEN JOSÉ ALVES DE ASSIS \\ UNIVERSIDADE FEDERAL DO PARÁ, BRASIL \\ ANA Claudia Moreira ALMEIDA Verdu \\ UNIVERSIDADE ESTADUAL PAULISTA - BAURU, BRASIL
}

\begin{abstract}
RESUMO
Este estudo buscou verificar o efeito do procedimento de Instrução com Múltiplos Exemplares (MEI) sobre habilidades verbais de falante e de ouvinte em três crianças de 8 a 12 anos com deficiência auditiva pré-lingual e implante coclear, utilizando três conjuntos de estímulos não-convencionados. Os repertórios-alvo do ensino foram: ecoico, discriminação auditivo-visual e tato. A Fase 1 consistiu na avaliação inicial dos repertórios de falante (tato e textual) e ouvinte (discriminação condicional auditivovisual). A Fase 2 estabeleceu treino de ouvinte encadeado com palavra ditada pela experimentadora aos estímulos visuais apresentados como modelo e com exigência de resposta ecoica dos participantes, além do treino de falante (tato) com o Conjunto de Estímulos 1. Na Fase 3 foram realizados treino de ouvinte e de falante utilizando-se o procedimento de MEI com o Conjunto de Estímulos 2. A Fase 4 consistiu em avaliação da integração dos repertórios após o MEI. A Fase 5 consistiu na reaplicação da Fase 3 com o Conjunto de Estímulos 3. Em seguida, os testes da Fase 1 foram reaplicados. Os participantes mostraram aquisição das discriminações auditivo visuais (ouvinte) e aumentos sistemáticos nos repertórios de ecoico e tato (falante) com estímulos específicos nas fases de ensino. No pós-teste mantiveram $100 \%$ de ecoicos corretos, e a porcentagem de tatos aumentou em relação ao pré-teste, mas ocorreram variações individuais (respectivamente 44,4, 55,5, e 77,7 \% para cada um dos três participantes). Discute-se a eficiência do procedimento de múltiplos exemplares na promoção da interdependência entre repertórios verbais para a população de implantados cocleares.
\end{abstract}

Palavras-chave: instrução com múltiplos exemplares, comportamento de ouvinte, comportamento de falante, deficiência auditiva pré-lingual, implante coclear.

\section{ABSTRACT}

This study aimed at verifying the effects of the Multiple Exemplar Instruction (MEI) procedures on verbal skills of speakers and listeners in three children from 8 to 12 years old with pre-lingual hearing impairment and cochlear implant by using three sets of non-conventional stimuli. The target-repertoires of the study were: echoic, auditory-visual discrimination, and tact. Phase 1 was the initial repertoire assessment of the speakers (tact and textual) and listeners (auditory-visual conditional discrimination). Phase 2 established the listener training chained by the experimenter's dictated word to the visual stimulus used as sample and the demand of echoic response from the participants, in addition to the speaker training (tact) with the Stimuli Set 1. In Phase 3 were performed the training of the listener and the speaker using the MEI procedure with the Stimulus Set 1 . Phase 4 consisted in the assessment of repertoire's integration after the MEI. Phase 5 consisted in reapplying Phase 3 with the Stimulus Set 3. Afterwards, Phase 1 tests were reapplied. The performance of the participants varied from $16 \%$ to $100 \%$ of accurate responses $t$ in the target-repertoire. The participants showed the acquisition of auditory-visual discrimination (listener) and systematic increase in the echoic and tact repertoires (speaker) with specific stimuli in the teaching phases. In the post-test 100\% of correct echoic was maintained and the percentage of tacts increased in comparison to the post- test, however some individual variations occurred (44,4; 55,$5 ; 77,7 \%$ respectively for each of the three participants). The efficiency of multiple exemplar procedures in promoting the interdependency between verbal repertoires for the population of people with cochlear implants is debated.

Key words: Multiple exemplar instruction, listener behavior, speaker behavior, pre-lingual hearing impairment, cochlear implant.

Esta pesquisa é parte da Tese de doutorado desenvolvida pela primeira autora, bolsista CNPq, junto ao Programa de Pós-Graduação em Teoria e Pesquisa do Comportamento (UFPA) sob orientação do segundo autor e co orientação da terceira autora. Os autores agradecem aos pesquisadores do Laboratório de Aprendizagem, Desenvolvimento e Saúde (UNESP-Bauru) pelas contribuições, em especial à Maria Carolina Yoshida pela assistência na coleta de dados. Grauben José Alves de Assis é bolsista de Produtividade em Pesquisa do CNPq. Ana Claudia Moreira Almeida Verdu é membro do Instituto Nacional de Ciência e Tecnologia sobre Comportamento, Cognição e Ensino, financiado pela FAPESP (Processo \#2008/57705-8) e pelo CNPq (Processo 573972/2008-7). Enviar correspondência para: Fabiane da Silva Pereira (fabpsi@ gmail.com). 
As pesquisas desenvolvidas no escopo da Análise do Comportamento com crianças implantadas cocleares têm fornecido protocolos de atuação sistematizada, baseada na previsão e controle do comportamento, buscando compreender como estas crianças inserem os sons às suas habilidades de falante e ouvinte. A maior parte das pesquisas documenta treino de exemplar único (do inglês Single Exemplar Instruction) com o treino de comportamento de ouvinte, com posterior avaliação do comportamento de falante, seja após cada fase de treino (Anastácio-Pessan, Almeida-Verdu, Bevilacqua, \& de Souza, 2015; AlmeidaVerdu, de Souza, Bevilacqua, \& Souza, 2009; Lucchesi, Almeida-Verdu, Buffa, \& Bevilacqua, 2015; Neves, Almeida-Verdu, Nascimento, \& Moret, 2013; Souza, Almeida-Verdu, \& 2013), ou somente após a conclusão de todas as fases de treino (Almeida-Verdu et al., 2008; Golfeto, 2010; Pereira, Lobato, Oliveira, Yamaguchi, Cordeiro, \& Galvão, no prelo).

Nos estudos que apresentaram uma rotina de treino de ouvinte seguido de testes do comportamento de falante (tato e leitura) observou-se variabilidade de desempenho entre os participantes; no entanto, constataram-se desempenhos consistentes nas habilidades de ouvinte e melhora gradual do comportamento de falante quando o delineamento envolvia sucessivos pós-testes (AnastácioPessan et al., 2015; Neves et al., 2013; e Lucchesi et al., 2015). Um aspecto desses estudos que merece investigação é se a fala em tarefas de tato de figuras passou a ter mais correspondência ponto a ponto com as convenções da comunidade verbal porque (a) a formação de classes (palavras ditadas, figuras e palavras impressas) transferiu o controle exercido pelas palavras impressas para as figuras (ex. de Souza, Hanna, de Rose, Fonseca, Pereira, \& Sallorenzo, 1997) ou se (b) os sucessivos testes de nomeação intercalados com as fases de ensino de relações auditivovisuais ofereceu condições para que os repertórios de ouvir e de falar passassem a ficar integrados, interdependentes. A resposta a essa questão é empírica.

Estudos têm evidenciado a independência funcional entre os operantes verbais (Cuvo \& Riva, 1980; Greer \& Ross, 2008) e estes resultados são ainda mais evidentes com a população de implantados cocleares, uma vez que aprender a ouvir não é suficiente para que o falar ocorra; e falar controlado por determinados estímulos (ex., textuais) não é condição para que ocorra na presença de outros estímulos (ex., figuras), sem que haja planejamento específico. A literatura em controle de estímulos e implante coclear, embora recente, fornece evidências empíricas suficientes sobre a independência funcional entre ouvir e falar (Almeida-Verdu et al., 2008; Battaglini, Almeida-Verdu, \& Bevilacqua, 2013). Nesses estudos, embora o comportamento de ouvir tenha sido estabelecido com precisão, não foram obtidos resultados positivos nos testes de tato. Uma pergunta desse campo de pesquisa tem sido quais seriam as condições para que os repertórios de falante e de ouvinte fossem estabelecidos de forma integrada em crianças pré-linguais com implante coclear (IC).

Durante o desenvolvimento infantil típico, os pais e/ou cuidadores nomeiam os objetos para os quais a criança olha; apontam para objetos e dizem seus nomes; e também reforçam diferencialmente topografias vocais que reproduzem sons parcialmente ou completamente parecidos a um estímulo verbal antecedente (Horne \& Lowe, 1996; Skinner, 1957), favorecendo o estabelecimento de ecoicos. Essas interações oferecem condições para o estabelecimento de habilidades de ouvinte e de falante.

Uma proposta metodológica recente de se produzir a interdependência de habilidades de ouvir e falar é pela estruturação da Instrução com Múltiplos Exemplares (MEI), do inglês Multiple-Exemplar Instruction (Greer, Yuan, \& Gautreax, 2005), na qual os estímulos auditivos funcionam, por um lado, como discriminativos para o comportamento de ouvinte e, por outro, para o comportamento de falante, pois apontar eventos e falar são controlados por um antecedente comum, o estímulo discriminativo (Horne \& Lowe, 1996). A partir desta aprendizagem, quando a criança estiver diante de novos objetos com função semelhante, sem a necessidade de um cuidador falar, pode emitir respostas verbais de falante e ouvinte correspondentes.

No MEI, o participante é exposto a uma rotação rápida entre diferentes topografias de resposta (por exemplo, ouvir baseado em seleção de estímulos e produção oral) e com sobreposição de controles de estímulos (por exemplo, palavra ditada controlando respostas de seleção e ecoico; figuras controlando respostas de seleção e de tato), com o objetivo de estabelecer a função conjunta e aprendizagem de múltiplas respostas a um estímulo. As pesquisas na área sugerem que as crianças que não demonstram comportamento de falante depois de aprender as relações de ouvinte podem ser capazes de fazê-lo depois do MEI (Fiorile \& Greer 2007; Greer et al., 2005; Greer \& Ross, 2008; Greer, Stolfi, \& Pistoljevic, 2007).

Com implantados cocleares não há trabalhos documentados utilizando a proposta de MEI de maneira sistemática. Contudo, nos trabalhos de Almeida-Verdu et al. (2009) e Souza, Almeida-Verdu \& Bevilacqua (2013) com palavras, e Golfeto e de Souza (2015) com sentenças, o repertório-alvo era o tato de figuras de objetos e de ações, respectivamente. Ainda que, com extensões de estímulos e estruturas de ensino diferentes, o ensino consistiu no comportamento ecoico combinado com discriminações auditivo-visuais; no entanto, os operantes não eram apresentados de modo rotativo nas tentativas. $\mathrm{O}$ delineamento demonstrou que o ensino de ecoico foi necessário para a verificação de tato. Os resultados demonstraram maior porcentagem de acertos no pós-teste de tato, se comparados com os dados obtidos no pré-teste.

Considerando as discussões levantadas e a necessidade de se verificar as condições necessárias para que os repertórios de ouvinte e falante possam ser integrados, isto é, tornar-se interdependentes em crianças com implante 
coclear, este trabalho teve como objetivo avaliar se o ensino estruturado por Exemplares Múltiplos (MEI) seria condição para o estabelecimento do repertório integrado de falante e ouvinte em crianças deficientes auditivas, pré-linguais, usuárias de IC.

\section{Participantes}

\section{MÉTODO}

Três crianças entre 8 e 12 anos, com experiência auditiva com IC igual ou superior a seis anos, participaram do estudo. Duas crianças estavam matriculadas no Ensino Fundamental I e uma no Ensino Fundamental II. Os resultados do teste PPVT - Peabody Picture Vocabulary Test, Edition IV (Dunn \& Dunn, 2015) aplicado mediante tradução das palavras, forneceram a idade linguística a partir de testes de reconhecimento auditivo que, para todos os participantes, mostrou-se inferior à idade cronológica. Todos os participantes tinham experiência em pesquisas em controle de estímulos. Suas principais características estão listadas na Tabela 1.

Tabela 1

Principais Características dos Participantes: Idade, Tempo de Implante, Nivel de Escolaridade e Idade Equivalente.

\begin{tabular}{ccccc}
\hline Participante & Idade & Tempo de IC & Escolaridade & PPVT \\
\hline JAN & $8 \mathrm{a} 11 \mathrm{~m}$ & $6 \mathrm{a} 8 \mathrm{~m}$ & $2^{\circ}$ ano & $3 \mathrm{a} 6 \mathrm{~m}$ \\
SUE & $10 \mathrm{a} 5 \mathrm{~m}$ & $\begin{array}{c}\text { OD: } 9 \mathrm{a} \\
\text { OE: } 2 \mathrm{a}\end{array}$ & $5^{\circ}$ ano & $5 \mathrm{a} 7 \mathrm{~m}$ \\
EDU & $13 \mathrm{a} 11 \mathrm{~m}$ & $\begin{array}{c}\text { OD: } 1 \mathrm{a} 6 \mathrm{~m} \\
\text { OE: } 7 \mathrm{a}\end{array}$ & $6^{\circ}$ ano & $6 \mathrm{a} 3 \mathrm{~m}$ \\
\hline
\end{tabular}

OD: orelha direita

OE: orelha esquerda

Para a participação no estudo foi necessária a assinatura dos responsáveis legais pelos participantes em Termo de Consentimento Livre e Esclarecido (TCLE). Este projeto foi aprovado pelo Serviço de Apoio ao Ensino, Pesquisa e Extensão - Comitê de Ética em Pesquisa [SVAPEPE-CEP] sob o protocolo 106/2009.

\section{Equipamentos, ambiente experimental e registro de respostas}

Foi utilizado um computador da marca DELL Inspirion 14 2640, com tela de 14 polegadas, sistema operacional Windows 8.1, para a apresentação dos estímulos visuais e mouse para seleção dos estímulos. Para a programação das tarefas e registro das respostas dos participantes foi utilizado o software EAM V. 4.0.04 (Nerigo, Monteiro, \& Sarraf, 2010), que permitiu programar as tarefas, assim como editá-las e reprogramá-las de acordo com os objetivos e necessidades de cada fase do ensino.
Também foi utilizado o software POWER POINT (versão 2016) para apresentação de algumas tarefas.

As sessões foram realizadas na casa dos participantes, em uma sala com iluminação adequada, com cadeiras e mesas para o uso das crianças, onde permaneciam somente experimentador, um auxiliar de coleta e o participante. As vocalizações e as respostas emitidas pelos participantes foram registradas pelo software Camtasia Recorder 8 (Brian, 2014), que permite a gravação da tela do computador e do participante simultaneamente, além de gravação das produções orais durante as tarefas.

\section{Estímulos}

Estímulos discriminativos: foram apresentados estímulos auditivos e visuais. Os estímulos auditivos foram gravados em voz feminina adulta e armazenados no computador. Os estímulos visuais eram figuras e palavras escritas apresentadas na tela do computador, classificados como convencionados e não convencionados (pertencentes e não pertencentes ao cotidiano dos participantes, respectivamente). Os estímulos utilizados são mostrados na Figura 1.

Estímulos consequentes: Para o levantamento de possíveis reforçadores tangíveis e não tangíveis realizou-se entrevistas com os pais e com as crianças. A cada tentativa, respostas corretas eram seguidas por consequências programadas para acerto (gifs animados escolhidos pelos participantes) e respostas incorretas seguidas pelo escurecimento da tela por 3s. Ao final das sessões, independentemente do desempenho da criança, eram conduzidas atividades lúdicas entre pesquisadoras e participante com brincadeiras iniciadas pelo participante.

\section{Delineamento Experimental}

Cada participante iniciou e concluiu as fases do estudo independentemente do outro. Foram realizados testes dos repertórios-alvo ao longo do estudo para verificação dos efeitos do procedimento. Além disso, foi realizado um préteste de avaliação geral das habilidades de ouvinte e de falante, e ao fim do estudo, foi realizado um pós-teste para reavaliação geral das mesmas habilidades.

A variável dependente (VD) deste estudo foi a integração dos repertórios de falante e de ouvinte mensurados pela porcentagem de acertos em tarefas de produção oral (ecoico ou tato) e de seleção e pela congruência entrea essas porcentagens. A variável independente (VI) foi o ensino em Instrução de Múltiplos Exemplares (MEI) constituído por operantes de quatro topografias distintas (IdMTS, ecoico, AvMTS e tato) e de controle por estímulos distintos também (palavra ditada e figura). 


\begin{tabular}{|c|c|c|c|c|c|c|c|c|}
\hline & $\begin{array}{l}\text { Palavra } \\
\text { ditada }\end{array}$ & Figura & $\begin{array}{l}\text { Palavra } \\
\text { escrita }\end{array}$ & & $\begin{array}{c}\text { Palavra } \\
\text { ditada }\end{array}$ & Figura & $\begin{array}{l}\text { Palavra } \\
\text { escrita }\end{array}$ & \\
\hline \multirow{10}{*}{ 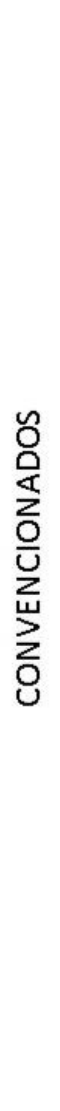 } & boca & & BOCA & \multirow{10}{*}{ 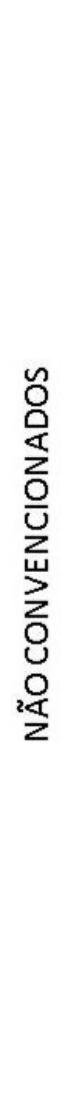 } & Tiba & & TIBA & \multirow{3}{*}{$\stackrel{\overbrace{}}{\mathrm{O}}$} \\
\hline & luva & & LUVA & & Zigo & & ZIGO & \\
\hline & sino & & SINO & & Pafe & & PAFE & \\
\hline & gato & & GATO & & Jeba & & JEBA & \multirow{3}{*}{$\stackrel{\text { O }}{\stackrel{\text { S }}{N}}$} \\
\hline & lata & & LATA & & Pito & & PITO & \\
\hline & mala & & MALA & & Lila & & LILA & \\
\hline & pato & & PATO & & Duca & & DUCA & \multirow{3}{*}{$\stackrel{\text { O }}{\stackrel{\rho}{2}}$} \\
\hline & tatu & & TATU & & Fado & & FADO & \\
\hline & Vela & c. & VELA & & Befi & & BEFI & \\
\hline & Vaca & & VACA & & & & & \\
\hline
\end{tabular}

Figura 1. Estímulos utilizados no estudo.

\section{Procedimento}

O procedimento de ensino foi estruturado por Exemplares Múltiplos (MEI), de acordo com Greer e Longano (2010). A Tabela 2 apresenta a ordem de apresentação dos tipos de tentativas e as principais características das fases deste estudo.

Fase 1 - Pré-teste: Avaliação do repertório de entrada. O Pré-teste avaliou as respostas de ouvinte baseadas em seleção (AvMTS) e de falante (ecoica, tato e textuais), diante de estímulos convencionados e não convencionados. As respostas de falante e de ouvinte eram avaliadas em uma mesma tentativa, de acordo com Souza et al. (2013).

O pré-teste tinha 38 tentativas, apresentadas em dois blocos (Bloco 1 e Bloco 2). O Bloco 1, com 19 tentativas, avaliou os comportamentos de ouvinte, ecoico e tato, com apresentação única de cada estímulo-modelo. Para manter a participação das crianças ao longo do teste sem reforçamento e evitar os efeitos prejudiciais deste sobre o repertório, foi apresentada consequência programada para as respostas corretas em $25 \%$ das tentativas com estímulos convencionados, isto é, em quatro tentativas na sessão. Não havia procedimento de correção de respostas incorretas.

Inicialmente, a criança recebia a instrução da experimentadora: "você ouvirá um som, depois que ouvir, repita o que ouviu e aponte para a figura que combina com o som" e então a sessão experimental tinha início. Uma tentativa iniciava com a apresentação de um estímulomodelo auditivo via alto-falantes do computador e, simultaneamente, um quadrado laranja apresentado na tela do computador. O estímulo-modelo auditivo era repetido a cada $5 \mathrm{~s}$ e o participante poderia ouvi-lo quantas vezes considerasse necessário antes de responder. A interrupção do som era conduzida pelo participante ao selecionar o quadrado laranja apresentado simultaneamente ao som. Após a apresentação do estímulo auditivo, a experimentadora solicitava ao participante a repetição do que ouviu (avaliação do ecoico); após a produção oral do participante, este selecionava o quadrado laranja, que após a seleção, desaparecia e dois estímulos visuais - figuras - eram apresentados na tela do computador, sendo apenas um deles 
correspondente ao modelo ditado. Era solicitado ao participante que apontasse para a figura relacionada ao estímulo-modelo auditivo (avaliação do comportamento de ouvinte). Após a seleção (apontar na tela), a experimentadora solicitava ao participante que falasse o nome da outra figura apresentada (avaliação de tato) através da instrução "qual é o nome deste outro?".

Tabela 2

Fases do Procedimento, Função de Cada Fase, Estímulos, Repertórios Alvo e Critério.

\begin{tabular}{|c|c|c|c|c|}
\hline Fase & Finalidade & Estímulos & Repertórios-alvo & Critério \\
\hline 1 & Pré-teste & $\begin{array}{l}\text { convencionados e não- } \\
\text { convencionados }\end{array}$ & Seleção, ecoico, tato e textual & - \\
\hline 2 & $\begin{array}{c}\text { Linha de base } \\
\text { e teste }\end{array}$ & $\begin{array}{c}\text { Conjunto } 1 \\
\text { (Tiba, Zigo e Pafe) }\end{array}$ & $\begin{array}{c}\text { IdMTS+ palavra ditada pela (tato) } \\
\text { experimentadora e ecoico } \\
\text { Avaliação de AvMTS e Tato }\end{array}$ & $\geq 90 \%$ \\
\hline 3 & MEI & $\begin{array}{c}\text { Conjunto } 2 \\
\text { (Jeba, Pito, Lila) }\end{array}$ & IdMTS; ecoico; AvMTS; tato & $\geq 90 \%$ \\
\hline 4 & Teste & $\begin{array}{c}\text { Conjunto } 1 \\
\text { (Tiba, Zigo, Pafe) }\end{array}$ & AvMTS e Tato & $\geq 90 \%$ \\
\hline 5 & $\begin{array}{c}\text { Treino de } \\
\text { ouvinte e teste }\end{array}$ & $\begin{array}{c}\text { Conjunto } 3 \\
\text { (Duca, Fado, Befi) }\end{array}$ & $\begin{array}{c}\text { IdMTS+ palavra ditada pela (tato) } \\
\text { experimentadora e ecoico } \\
\text { Avaliação de AvMTS e Tato }\end{array}$ & $\geq 90 \%$ \\
\hline 6 & Pós-teste & $\begin{array}{c}\text { Convencionados e não- } \\
\text { convencionados }\end{array}$ & Seleção, ecoico, tato e textual & - \\
\hline
\end{tabular}

As combinações entre os estímulos de comparação sempre apresentavam um estímulo convencionado e um não convencionado. Somente depois da avaliação dos três operantes (ouvinte baseado em seleção, ecoico e tato) é que o estímulo correspondente à palavra ditada poderia ser selecionado com o mouse, o que dava acesso a uma nova tentativa.

O Bloco 2 avaliou o comportamento textual. Era composto por 19 tentativas com apresentação única de cada palavra testada. O bloco de tentativas iniciava com uma instrução para leitura "o que está escrito?" seguida da apresentação das palavras de forma sucessiva e em sequência aleatória.

Fase 2 - Ensino de repertório de ouvinte e ecoico e avaliação do tato com Conjunto 1. Com o objetivo de estabelecer o repertório mínimo de ouvinte e de ecoico, para verificação se esses dois repertórios seriam condição para a integração dos repertórios de ouvinte e falante, realizou-se treino de comportamento de ouvinte em tarefas de discriminação condicional por identidade (IdMTS), com apresentação de um estímulo modelo composto, que consistia na apresentação da figura simultaneamente ao modelo auditivo (o nome da figura) apresentado pela experimentadora e solicitação da repetição pelo participante (ecoico - treino de comportamento de falante). Eram realizadas 18 tentativas, seis para cada uma das três relações de identidade apresentadas envolvendo apenas os estímulos não-convencionados. $\mathrm{O}$ critério para encerramento do treino de IdMTS com estímulo composto foi um desempenho superior a $90 \%$ de acertos em três sessões consecutivas.
Uma tentativa de ensino se configurou em três passos: diante da apresentação de uma figura como estímulo-modelo na tela do computador e a palavra correspondente ditada pela experimentadora, o participante deveria repetir o estímulo auditivo, emitindo um ecoico e, em seguida, responder como ouvinte, selecionando o estímulo visual correspondente ao auditivo. A resposta ecoica do participante era modelada com dicas orofaciais em caso de distorções fonéticas e semânticas; a modelagem de ecoico poderia ocorrer em até três tentativas.

Em seguida, eram realizados dois testes: testes dos repertórios de ouvinte, via tentativas de discriminação condicional auditivo-visual (AvMTS), e de falante, em tentativas de Tato. Os testes eram conduzidos separadamente, com 12 tentativas cada, quatro para cada estímulo, apresentados randomicamente. Em caso de desempenho abaixo de $90 \%$ de acertos em algum dos testes, o participante era exposto à Fase 3, estruturada de acordo com o procedimento de MEI.

Fase 3 - Instrução com Múltiplos Exemplares (MEI) com Conjunto 2. A Fase 3 apresentava tentativas de ensino dos repertórios de ouvinte pela sobreposição do tato da experimentadora em tentativas de matching de identidade (IdMTS), assim como na Fase 2; foram acrescentadas tentativas de treino de ouvinte (AvMTS) e de treino de falante (tato) com três novos estímulos (Conjunto 2). De acordo com a proposta de rotatividade de operantes no ensino estruturado por múltiplos exemplares, uma sessão expunha o participante a quatro repertórios aleatorizados em 36 tentativas, sendo 12 de IdMTS com estímulo composto 
(figura + palavra ditada) + ecoico, 12 para AvMTS e 12 para treino de tato. Eram apresentadas quatro tentativas para cada estímulo. $\mathrm{O}$ critério para que o participante avançasse para a próxima fase era de pelo menos $90 \%$ de respostas corretas.

Fase 4 - Teste de integração dos repertórios de ouvinte e de falante com o Conjunto 1. Esta fase teve como objetivo verificar se, após a aquisição de repertórios de ouvinte e falante em um ensino estruturado por MEI com estímulos do Conjunto 2, o participante apresentaria os repertórios de falante e ouvinte com precisão com estímulos do Conjunto 1, com os quais o participante não havia alcançado critério de aprendizagem. O teste tinha as mesmas configurações dos testes de AvMTS e Tato do participante. Se a porcentagem de acertos fosse superior a $90 \%$ de acertos, o participante era exposto à Fase 5, com um terceiro conjunto de estímulos.

Caso o participante não demonstrasse o tato com os estímulos do Conjunto 1, era realizada uma nova sessão de IdMTS com estímulo composto (figura + palavra ditada) + ecoico para expor o participante novamente aos nomes dos estímulos (IdMTS + palavra ditada) e à oportunidade de emitir a produção oral (ecoico) com critério de pelo menos $90 \%$ de acertos em duas sessões consecutivas ou $100 \%$ de acertos em uma sessão. Após o treino, o participante era exposto aos testes de tato novamente. Caso ainda não demonstrasse o repertório de tato desses estímulos, era exposto ao treino com as características de MEI com um terceiro conjunto de estímulos, seguida do teste de avaliação novamente, conforme Fase 5.

Fase 5 - Treino de ouvinte e ecoico seguido de teste de tato com Conjunto 3. O objetivo desta fase era obter os repertórios de ouvinte e de falante de forma integrada, com um terceiro conjunto de estímulos, funcionando, assim, para a confiabilidade dos dados das fases anteriores. Os participantes foram expostos ao ensino por identidade visual (IdMTS) com estímulo composto (figura e palavra ditada pela experimentadora) sobreposto e solicitação do comportamento ecoico pelos participantes, conforme Fase 2. Após a obtenção do critério de acertos de pelo menos $90 \%$ de acertos, os participantes foram avaliados nos testes de ouvinte (AvMTS) e falante (Tato). Se apresentassem desempenho consistente, este resultado confirmaria a emergência da integração dos repertórios de falante e ouvinte.

Fase 6 - Pós-teste. Nesta fase, o pré-teste aplicado na Fase 1 foi reaplicado para avaliação do efeito do procedimento sobre os repertórios avaliados.

\section{RESULTADOS}

Os resultados dos participantes ao longo do estudo na realização de tarefas cujo objetivo foi treinar as habilidades de ouvinte e de falante (ecoico e tato) estão apresentados na Figura 2, de acordo com as fases às quais foram submetidos.
No Pré-teste (Fase 1), todos os participantes apresentaram desempenho acurado na avaliação do comportamento textual, demonstrando controle pelas unidades textuais mínimas que compõem as palavras. O comportamento de ouvinte baseado em seleção também foi acurado, tanto com estímulos convencionados quanto não convencionados.

Esse resultado no comportamento de ouvinte no pré-teste era possível e esperado, uma vez que as crianças poderiam responder por exclusão quando o estímulo modelo era uma palavra não convencionada e as comparações eram uma figura convencionada e outra não convencionada. Somente SUE apresentou desempenho abaixo do critério (33\% de acertos) de respostas corretas no teste de ouvinte com estímulos não convencionados. $\mathrm{Na}$ avaliação das respostas ecoicas e de tatos, todos apresentaram baixas porcentagens de acertos com os estímulos não convencionados, apresentando variação entre $20 \%$ e $65 \%$ de acertos e desempenho nulo em tato para estímulos não convencionados. A correspondência ponto-a-ponto em tarefas de ecoico com estímulos convencionados foi de $22,2 \%$ de acertos para o participante JAN (sons PITO e FADO), 66,6\% de acertos para SUE (JEBA, ZIGO, PITO, BEFI, TIBA e DUCA) e de $44,4 \%$ de acertos para EDU (ZIGO, PITO, TIBA e FADO). Considerando esse desempenho no repertório de falante, o ensino prosseguiu.

$\mathrm{Na}$ Fase 2, todos os participantes atingiram o critério estabelecido de linha de base de ouvinte. As respostas ecoicas de todos os participantes apresentaram frequência de correspondência ponto-a-ponto crescente ao longo do treino de linha de base. Nos testes de repertórios não treinados, AvMTS (ouvinte) e tato (falante), todos os participantes apresentaram abaixo de $90 \%$ de acertos em pelo menos um dos testes. Com base nestes dados, todos os participantes foram expostos ao MEI.

Com a exposição dos participantes ao ensino estruturado por MEI (Fase 3), todos os participantes chegaram à precisão nos comportamentos de ouvinte e falante (ecoico e tato), após duas (SUE) e três (JAN e EDU) revisões desse ensino.

$\mathrm{Na}$ Fase 4, os participantes JAN e EDU apresentaram $100 \%$ de respostas corretas no teste de tato e $83,3 \%$ e $75 \%$ respectivamente no teste de ouvinte. A participante SUE apresentou $16,6 \%$ de respostas corretas no teste de ouvinte e, por um erro da experimentadora, não foi exposta ao teste de tato. Para esta participante foi realizado um procedimento de ensino adicional, de revisão do ensino conduzido na Fase 3, seguido de nova exposição às condições da Fase 4 quando o desempenho em tato chegou a $90 \%$ de acertos.

Na Fase 5, conduzida com estímulos totalmente novos (Conjunto 3), os participantes EDU e JAN apresentaram desempenho acurado no comportamento de falante e ouvinte, e a participante SUE alcançou o critério no componente de falante $(91,6 \%)$ e repertório de ouvinte próximo ao critério de aprendizagem, $83,3 \%$ de acertos. 


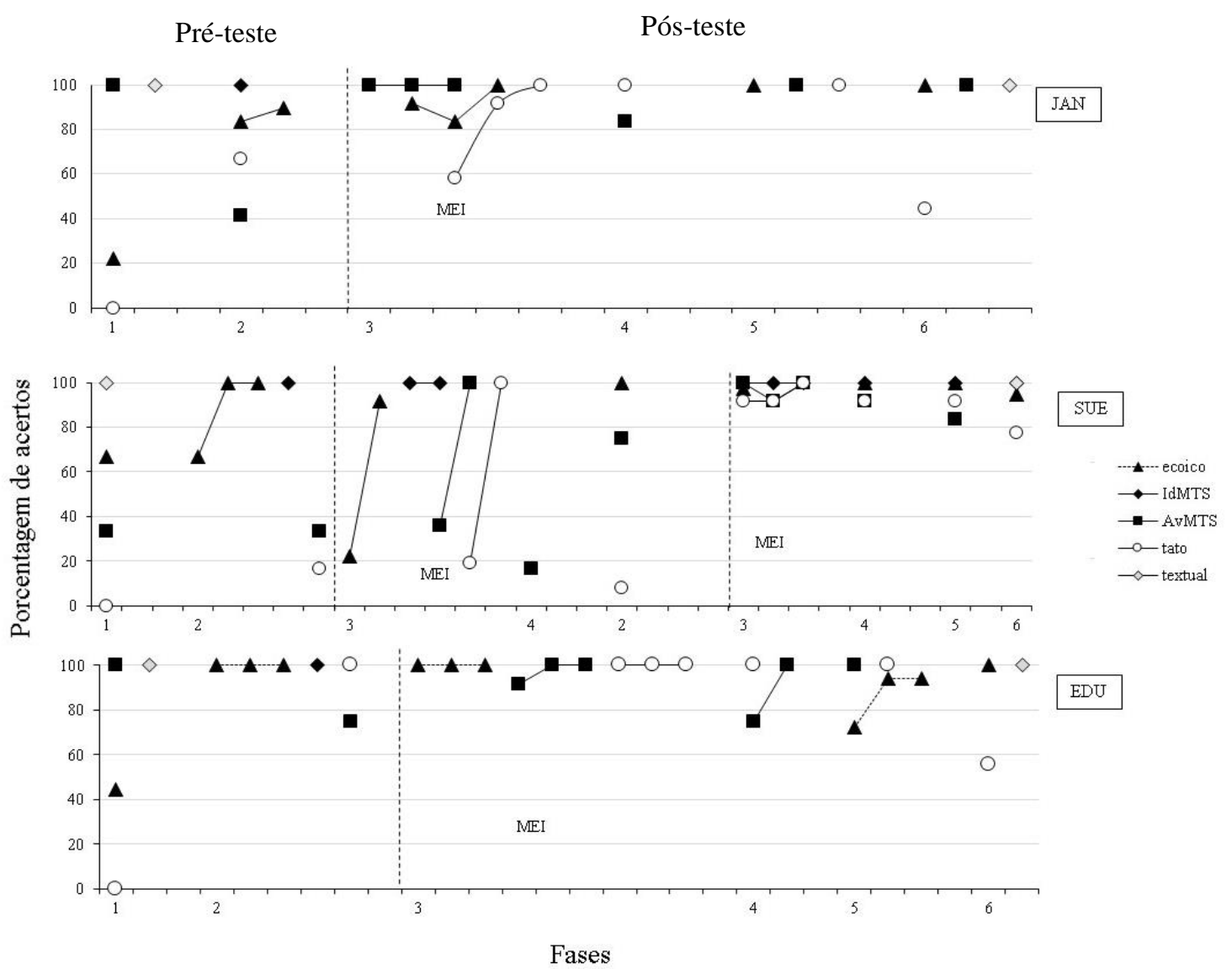

Figura 2. Desempenho dos participantes ao longo das fases de teste e ensino em relação aos repertórios-alvo com os estímulos não convencionados. A linha tracejada indica o início do procedimento MEI.

No Pós-teste (Fase 6), todos os participantes mantiveram os desempenhos de leitura com a porcentagem de acertos idênticas à avaliação do Pré-teste. Além disso, apresentaram melhorias em seus desempenhos na tarefa de ouvinte baseado em seleção, ecoico e de tato, com porcentagem de acertos superior à do Pré-teste. Enfatizando o desempenho dos participantes na tarefa de tato, ainda que todos tenham apresentado desempenhos superiores comparados aos obtidos no pré-teste, observou-se diminuição na porcentagem de acertos se comparados aos resultados das fases anteriores. $O$ desempenho dos participantes JAN, SUE e EDU, na avaliação do tato foi, respectivamente, $44,4 \%, 77,7 \%$ e $55,5 \%$ de acertos.

\section{DISCUSSÃO}

O objetivo de estabelecer a integração dos repertórios de ouvinte e falante via ensino estruturado com Múltiplos Exemplares (MEI) em crianças com deficiência auditiva pré-lingual, usuárias de implante coclear, foi alcançado.

A literatura tem discutido quais as condições que produzem transferência no controle das relações que envolvem o comportamento de ouvinte sobre as relações que envolvem o comportamento de falante em crianças usuárias de implante coclear. Parte dos procedimentos documentados até o momento tem apresentado como resultados habilidades de ouvinte mais acuradas do que as de falante (Battaglini et al., 2013). Outra parcela de estudos (Lucchesi et al., 2015; Anastacio Pessan et al., 2015), com melhores resultados em habilidades de falante tem se apoiado no estabelecimento do controle por unidades mínimas (por procedimentos de seleção de sílabas ou de composição de palavras por sílabas) em tarefas que se alternam com sondas de tato. Intercalar tarefas de seleção de palavras com tarefas de composição de palavras ou nomeação de figuras confere ao ensino uma estrutura com múltiplos exemplares de estímulos (palavra ditada, figura) e de respostas (seleção, composição e produção oral), de acordo com a proposta de MEI de Greer e Ross (2008). No entanto, essa hipótese não havia sido testada isoladamente. Os dados encontrados parecem mostrar o caminho ainda não explorado sobre a produção acurada de habilidades de falante e ouvinte nesta população.

Embora os participantes tenham concluído todas as fases deste estudo, os resultados da avaliação ecoica no préteste, com um grande número de erros tanto em sons convencionados quanto em sons não convencionados, 
levantam a discussão de alguns aspectos: a) o aparato e ambiente experimental utilizado para coleta de dados deve ser controlado, pois crianças com IC podem ter dificuldade de discriminar sons em ambientes ruidosos (Pereira et al., no prelo) por um lado e, por outro, uma única tentativa de apresentação da estimulação sonora pode não ser suficiente para que a palavra seja discriminada e repetida o que estabelece b) a necessidade de ensino de pré-requisitos.

Os baixos desempenhos em tarefas de tato no préteste eram esperados, uma vez que as figuras não eram representativas ou convencionadas; no entanto, os baixos desempenhos de ecoico não eram esperados pelo tempo de IC dos participantes, que variou de 6 a 9 anos, e consequentemente implica em experiências auditivas diversas na comunidade verbal ouvinte-falante; no entanto, os dados evidenciam o quanto ainda apresentaram dificuldades em seus repertórios ecoicos. O ecoico é um elemento chave para a integração das habilidades de ouvinte e falante (Horne \& Lowe, 1996).

O desempenho dos participantes ao longo da exposição às fases desse estudo indicou maior controle auditivo e adicionalmente produções ecoicas com maior correspondência. Além disso, no pós-teste os participantes melhoraram as porcentagens de acertos em ecoico e esta foi estendida para tato com estímulos novos. Esses resultados sugerem que o ensino estruturado por MEI seja capaz de integrar os repertórios de ouvinte ou de falante com mais acurácia, confirmando os dados anteriormente descritos na literatura com participantes com Transtorno do Espectro Autista, obtidos por Fiorele e Greer (2007), Greer et al. (2005), Greer e Ross (2008) e Greer et al. (2007).

Desta forma, a emissão de ecoicos tem função de ocasionar uma condição em que o próprio objeto entre em contato direto com o controle do comportamento verbal da criança. Essa argumentação é consistente com observações feitas pela audiologia e reabilitação pelo implante coclear, demonstrando que o comportamento de repetir o que se ouve da fala de outras pessoas (ecoar) e o que se ouve da própria fala (auto ecoar) se constitui em um repertório propulsor do desenvolvimento do comportamento de ouvinte e de falante (Fagan, 2015).

A literatura da audiologia, de maneira geral, reconhece a importância da repetição no desenvolvimento da fala de crianças com e sem implante coclear (Fagan, 2015). Embora a literatura registre que uma melhora nos desempenhos ecoicos, de ouvinte baseado em seleção melhora também o comportamento de falante em tarefas de tato, o real efeito do ensino de ecoico requer investigações mais sistemáticas em crianças com implante coclear, pois os resultados apresentados por Almeida-Verdu et al. (2009) e Souza et al., (2013) demonstraram que essa melhora é em relação ao repertório inicial, mas as condições para que seja emitido com precisão requer investigações. Nesses estudos o ensino de ecoico e de comportamento de ouvinte foi realizado dentro de uma estrutura de ensino por únicos exemplares. No presente estudo, os resultados mais integrados de ouvinte e de falante podem ser em decorrência do uso da estrutura de MEI.

Por exemplo, quando a criança já tem estabelecido um repertório de ecoicos, o estímulo auditivo "bola" poderá estabelecer ocasião para uma vocalização com correspondência ponto-a-ponto em relação ao estímulo verbal produzido pelo falante, e que ainda poderá ocasionar o próprio comportamento de ouvinte da criança em relação à bola (olhando-a ou pegando-a). Este encadeamento de respostas é uma condição para a criança se tornar uma falante-ouvinte em relação ao seu próprio estímulo verbal "bola", embora o elemento inicial desta cadeia seja a vocalização de outros (Greer \& Ross, 2008; Horne \& Lowe, 1996).

Um aspecto importante no ensino do repertório ecoico realizado nesta pesquisa foi a necessidade de fornecimento de dicas orofaciais no procedimento de correção. Este dado é coerente com o apresentado por Almeida-Verdu et al. (2009), em que dois participantes necessitaram do ensino do ecoico com pistas orofaciais para refinar a topografia vocal envolvida na avaliação de tato. Portanto, para esta população, a vocalização, quando tida como repertório-alvo de ensino sistemático, pode produzir melhoras no desempenho de falante e ouvinte (AlmeidaVerdu et al., 2009; Greer \& Ross, 2008).

Destaca-se os resultados obtidos com todos os participantes na Fase 5, na qual todos os participantes apresentaram desempenho superior a $80 \%$ de acertos nos testes de ouvinte e falante. Embora a participante SUE não tenha atingido o critério de $90 \%$ no teste de ouvinte, seus resultados fornecem indícios da efetividade do procedimento de MEI com esta população. Ademais, o desempenho abaixo do critério de SUE pode sugerir ainda um provável déficit no repertório de ouvinte da participante, o que caracteriza uma condição pré-requisito para o desenvolvimento completo da integração do repertório de falante e ouvinte (Greer \& Ross, 2008). Neste caso, torna-se necessário o desenvolvimento de procedimentos específicos para a instalação do repertório de ouvinte bem discriminado e de modelagem e refinamento do repertório de falante, e por fim, procedimentos que treinem as habilidades de modo conjunto, como a proposta deste estudo. Dentre os procedimentos de ensino de habilidades de ouvinte e de falante especialmente importantes para essa população está o treino do comportamento ecoico (Souza et al., 2013).

Algumas limitações encontradas neste estudo podem ser pontuadas: o uso de palavras com fonemas de mesmo ponto de articulação (p.ex. "lila") devem ser evitadas, pois neste estudo foi uma condição geradora de vocalizações sem correspondência ou correspondência parcial em todos os participantes. É necessário controle desta variável em um contexto de pesquisa, para se verificar o efeito do procedimento sobre o responder-alvo (Golfeto, 2010).

A forma como o pré-teste foi apresentado gerou desempenho por exclusão nos participantes em seus 
repertórios de seleção, que diante de um som não convencionado e duas figuras de comparação (uma convencionada e uma não convencionada) responderam à figura de comparação não convencionada por terem uma história extra experimental com as figuras convencionadas. No entanto, nas avaliações dos repertórios ecoicos, a história com os nomes dos estímulos convencionados não foi suficiente para produzir ecoicos com correspondência a sons não convencionados. Este modelo de tentativa deve ser evitado para que a verificação do repertório com os estímulos experimentais seja mais fidedigna possível, ainda que os baixos repertórios em ecoico devam ser destacados em contraposição ao desempenho por exclusão baseado em seleção.

Futuras pesquisas podem controlar essa variável de diferentes formas, seja fazendo a edição dos sons utilizados limpando ruídos, seja realizando as tarefas em salas com isolamento acústico ou usando o sistema de frequência modulada (disponibilizado pelo Sistema Único de Saúde) para aperfeiçoar a detecção do sinal sonoro.

Sugere-se que estudos futuros adotem delineamentos experimentais para verificar o efeito do MEI sobre a emergência de respostas verbais novas. Por exemplo, no caso de um delineamento de linha de base múltipla entre participantes, após a verificação de uma linha de base estável, mas com baixas porcentagens de acertos em repertório de tato, por exemplo, em pelo menos três participantes, os participantes podem ser expostos às seguintes condições experimentais: (a) enquanto um participante recebe o ensino rotativo de ouvinte, ecoico e tato com um conjunto de estímulos e verifica-se se o tato emerge com outro conjunto de estímulos após o ensino de ouvinte, (b) os demais participantes recebem somente o ensino de ouvinte; (c) observados resultados positivos no primeiro participante, os demais poderiam ser expostos, um a um, ao ensino rotativo e verificar se, sob as mesmas condições que o primeiro participante foi exposto e somente após elas, resultados semelhantes seriam observados. A comparação seria do efeito que o procedimento de MEI tem para cada participante que o recebeu em momentos diferentes (Cozby, 2003).

Outra possibilidade é o delineamento de sondas múltiplas, que consiste na avaliação dos repertórios-alvo antes e após a instrução do MEI, assim como descreveram Nuzzolo-Gomes e Greer (2004). Naquele estudo, nenhum dos participantes apresentava tatos ou mandos com três conjuntos de estímulos constituídos de substantivos + adjetivos. Foram ensinados tatos (ou mandos) para um conjunto de estímulos enquanto recebiam sonda em mando (ou tato), o operante não ensinado e nenhum estudante emitiu o operante não ensinado. O ensino por MEI expôs os participantes ao ensino de tato e mando alternadamente até a precisão com o segundo conjunto de estímulos. Finalmente, foram ensinados tatos (ou mandos) com o terceiro conjunto de estímulos e o operante não ensinado emergiu. No delineamento de sondas múltiplas os efeitos da variável independente são avaliados pela tomada de medidas repetidas da variável dependente ao longo de todas as fases do procedimento. No caso do estudo de Nuzzolo-Gomes e Greer, o operante emergente (seja de tato ou de mando, a depender da condição a qual o participante foi exposto) foi avaliado após o ensino por SEI (ensino de tato ou de mando, apenas) e após o ensino por MEI (ensino de tato e mando em rotatividade). $\mathrm{O}$ resultado desejado, qual seja, a emergência de um operante, foi observada somente após o participante ter sido exposto à condição de MEI.

Se a condição de ensino por MEI se constituirá em uma rota para que seja observada a interdependência entre repertórios de falante e de ouvinte em crianças com implante coclear, com potencial de aplicação em condições clínicas assim como já tem demonstrado os resultados consolidados quando a rota é por relações de equivalência (AnastácioPessan et al, 2015; Lucchesi et al, 2015; Almeida-Verdu \& Golfeto, 2016), ainda requer evidência empírica. Os resultados observados no presente estudo convidam para que novas pesquisas sejam realizadas com delineamentos mais bem controlados que repliquem os resultados de emergência de um operante verbal não treinado após o ensino por MEI com criança com implante coclear mais recente e crianças com idade cronológica mais tenra e, consequentemente, com menor experiência auditiva.

\section{REFERENCIAS}

Almeida-Verdu, A. C. M., \& Golfeto, R. M. (2016). Stimulus control and verbal behavior: (in)dependent relations in populations with minimal verbal repertoires. In J. C. Todorov (Ed.), Trends in Behavior Analysis (187226). Brasília, DF.: Technopolitik

Almeida-Verdu, A. C. M., de Souza, D. G., Bevilacqua, M. C., \& Souza, F. C. (2009). Imitação vocal e nomeação de figuras em deficientes auditivos usuários de implante coclear: estudo exploratório. Revista Brasileira de Análise do Comportamento, 5(1), 63-78.

Almeida-Verdu, A. C. M., Huziwara, E. M., de Souza, D. G., de Rose, J. C., Bevilacqua, M. C., Lopes, J., \& McIlvane, W. J. (2008). Relational learning in children with deafness and cochlear implants. Journal of the Experimental Analysis of Behavior, 89(3), 407-424. doi: http://doi.org/10.1901/jeab.2008-89-407.

Anastácio-Pessan, F. L., Almeida-Verdu, A. C. M., Bevilacqua, M. C., \& de Souza, D. G. (2015). Usando o paradigma de equivalência para aumentar a correspondência na fala de crianças com implante coclear na nomeação de figuras e na leitura. Reflexão $e$ Crítica, 28(2), 365-377. doi: https://dx.doi.org/10.1590/1678-7153.201528217

Battaglini, M. P., Almeida-Verdu, A. C. M., \& Bevilacqua, M. C. (2013). Aprendizagem via exclusão e formação de classes de equivalência em crianças com deficiência auditiva e implante coclear. Acta Comportamentalia: Revista Latina de Análisis de Comportamiento, 21(1) 2035. Recuperado de 
http://www.redalyc.org/articulo.oa?id=274526149004

Brian, S. (2014). Camtasia Studio 8. The Charleston $\begin{array}{llll}\text { Advisor, } & 16 & \text { (2), } & 19-21(3) .\end{array}$ http://dx.doi.org/10.5260/chara.16.2.19

Cozby, P.C. (2003). Métodos de Pesquisa em Ciências do Comportamento. São Paulo: Atlas.

Cuvo, A. J., \& Riva, M. T. (1980). Generalization and transfer between comprehension and production: a comparison of retarded and nonretarded persons. Journal of Applied Behavior Analysis, 13(2), 315-331.doi: http://doi.org/10.1901/jaba.1980.13-315.

de Souza, D. G., Hanna, E. S., de Rose, J. C., Fonseca, M. L., Pereira, A. B., \& Sallorenzo, L. H. (1997). Transferência de controle de estímulos de figuras para texto no desenvolvimento de leitura generalizada. Temas em Psicologia (Ribeirão Preto), 5(1), 33-46.

Dunn, L. M., \& Dunn, D. M. (2015). Peabody Picture Vocabulary Test: PPVT 4. Pearson.

Fagan, M. K. (2015). Why repetition? Repetitive babbling, auditory feedback, and cochlear implantation. Journal of Experimental Child Psychology,137, 125-136. doi: http://doi.org/10.1016/j.jecp.2015.04.005.

Fiorile, C. A., \& Greer, R. D. (2007). The induction of naming in children with no prior tact responses as a function of multiple exemplar histories of instruction. The Analysis of Verbal Behavior, 23 (1), 71-88.

Golfeto, R. M. (2010). Compreensão e produção de fala em crianças com surdez pré-lingual usuárias de implante coclear. Tese de Doutorado. São Carlos: Universidade Federal de São Carlos. Recuperado de http://www.ufscar.br/ecce/wp content/files_flutter/1280109367TeseRMGolfeto.pdf

Golfeto, R. M., \& de Souza, D. G. (2015). Sentence production after listener and echoic training by prelingual deaf children with cochlear implants. Journal of Applied Behavior Analysis, 48 (2), 363-75. doi: http://doi.org/10.1002/jaba.197

Greer, D., Stolfi, L., \& Pistoljevic, N. (2007). Emergence of naming in preschoolers: A comparison of multiple and single exemplar instruction. European Journal of Behavior Analysis, $8 \quad$ (2),109-131. Doi: http://dx.doi.org/10.1080/15021149.2007.11434278

Greer, R. D., \& Longano, J. (2010). A rose by naming: how we may learn how to do it. The Analysis of Verbal Behavior, 26(1), 73-106.

Greer, R. D., \& Ross, D. E. (2008). Verbal behavior analysis: inducing and expanding new verbal capabilities in children with language delays. Boston: Pearson Education.

Greer, R. D., Yuan, L., \& Gautreaux, G. (2005). Novel dictation and intraverbal responses as a function of a multiple exemplar history. The Analysis of Verbal Behavior, 21 (1), 99-116.

Horne, P. J., \& Lowe, C. F. (1996). On the origins of naming and other symbolic behavior. Journal of the Experimental Analysis of Behavior, 65(1), 185-241. doi: http://doi.org/10.1901/jeab.1996.65-185.

Lucchesi, F. D. M., Almeida-Verdu, A. C. M., Buffa, M. J. M. B., \& Bevilacqua, M. C. (2015). Efeitos de um programa de ensino de leitura sobre a inteligibilidade da fala de crianças usuárias de implante coclear. Psicologia: Reflexão e Crítica, 28(3), 500-510. doi: https://doi.org/10.1590/1678-7153.201528309.

Nerigo, G. S. JR., Monteiro, D., \& Sarraf, N. (2010). Desenvolvimento de software para apoio da aprendizagem em primatas e crianças com próteses auditivas e implantes cocleares. In: I Simpósio do Instituto Nacional de Ciência e Tecnologia sobre Comportamento, Cognição e Ensino, 2010, São Paulo.

Neves, A. J., Almeida-Verdu, A. C. M., Moret, A. M., \& Nascimento, L. T. (2013). Correspondence while reading and the designation of sentences in children with hearing disabilities who use pre-lingual cochlear implants. International Archives of Otorhinolaryngology, 17(1), 7.

Nuzzolo-Gomez, R., \& Greer, R. D. (2004). Emergence of untaught mands or tacts of novel adjective-object pairs as a function of instructional history. The Analysis of Verbal Behavior, 20, 63-76.

Pereira, F. S., Lobato, S. N. S., Oliveira, J. S. C., Yamaguchi, C. T., Barros, J. C. C., \& Galvão, O. F. (in press). Discriminação auditiva-visual em deficientes auditivos pós-linguais com implante coclear. Temas em Psicologia (Ribeirão Preto).

Skinner, B. F. (1957). Verbal Behavior. New York: Applenton-Century-Crofts.

Souza, F. C., Almeida-Verdu, A. C. M., \& Bevilacqua, M. C. (2013). Ecoico e nomeação de figuras em crianças com deficiência auditiva pré-lingual com implante coclear. Acta Comportamentalia: Revista Latina de Análisis de Comportamiento, 21(3), 325-339.

Submetido em 22/03/2016 Aceito em 03/11/2016 